\title{
FREE FLOATING THROMBUS OF COMMON CAROTID ARTERY CAUSING STROKE IN AN ANEMIC PATIENT AFTER ABDOMINAL SURGERY: CASE REPORT AND REVIEW OF LITERATURE
}

\footnotetext{
M. Emamikhah1, N. Mohebi2, M. Moghaddasi2, N. Yazdi2, M. Eslami2.

1Department of neurology- Rasool-e Akram hospital- Iran university of medical sciences, Tehran, Iran.

2Department of neurology- Rasool-e Akram hospital- Iran university of medical sciences, Tehran, Iran
}

\section{Background and Aims}

Free floating thrombosis (FFT) of carotid artery is a rare condition leading to stroke. Atherosclerosis is the most prevalent etiology. Non-atherosclerotic causes are even much scarcer. There is no unified consensus about diagnostic approach and management of FFT patients.

\section{Methods:}

We present a patient with FFT lacking any atherosclerotic disease and was managed successfully with a combined medical and surgical approach.

\section{Results:}

A 49-year-old male admitted for colostomy reversal. After surgery, physical examination revealed global aphasia and right hemiparesis. Risk factors regarding atherosclerosis or hypercoagulability state were unremarkable. Brain magnetic resonance imaging (MRI) showed multiple cortical acute infarctions. Duplex sonography (DS) of carotid arteries showed a large fresh floating thrombosis in left common carotid artery (CCA) confirmed by Computerized tomography angiogram (CTA). The comprehensive laboratory investigations were normal, except for transient anemia and thrombocytosis. Anticoagulation started and was continued after urgent surgery for 10 days followed by antiplatelet. There were no further complications in 3 months follow-up.
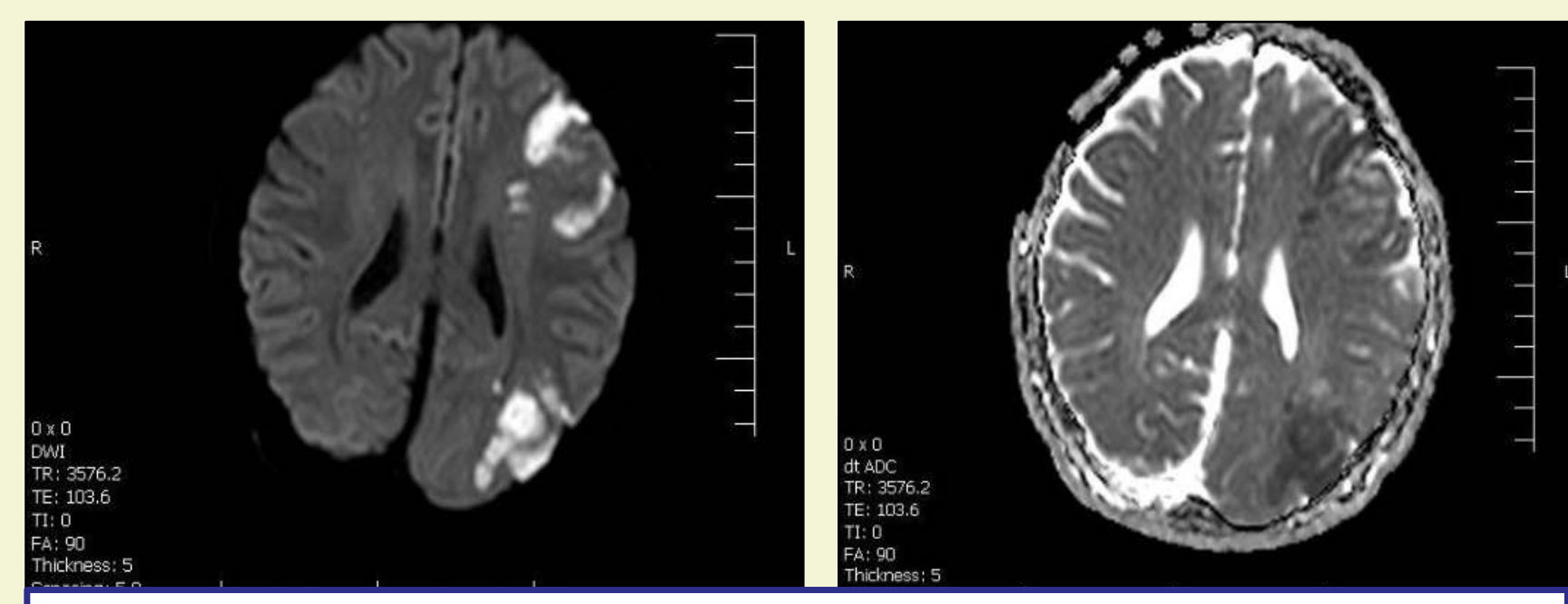

DWI (Left) and ADC map (Right) brain MRI. Hyper-intensities are evident in the left frontoparietal lobe with restriction in the DWI-ADC sequences, (acute infarction in left MCA territory)
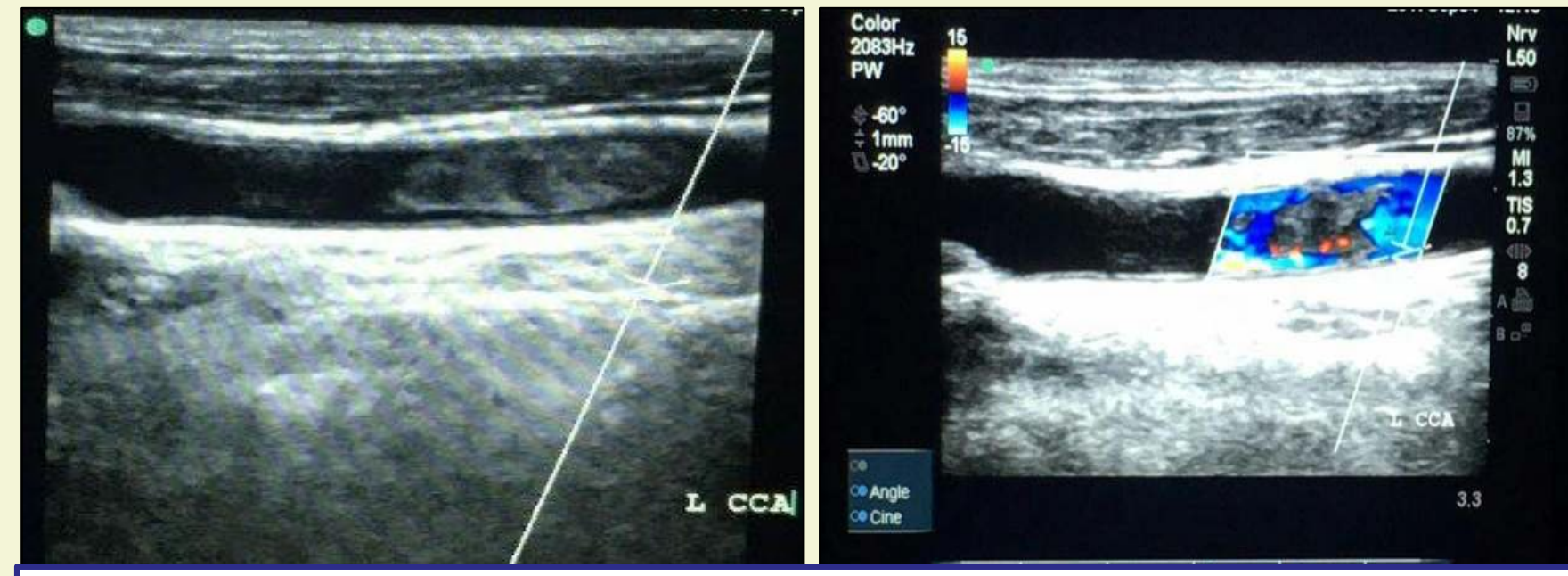

Left: B-mode duplex sonography of left common carotid artery showed an isoechoic intraluminal lesion compatible with fresh floating clot. Right: Turbulent and circumferential blood flow in the intraluminal thrombosis.
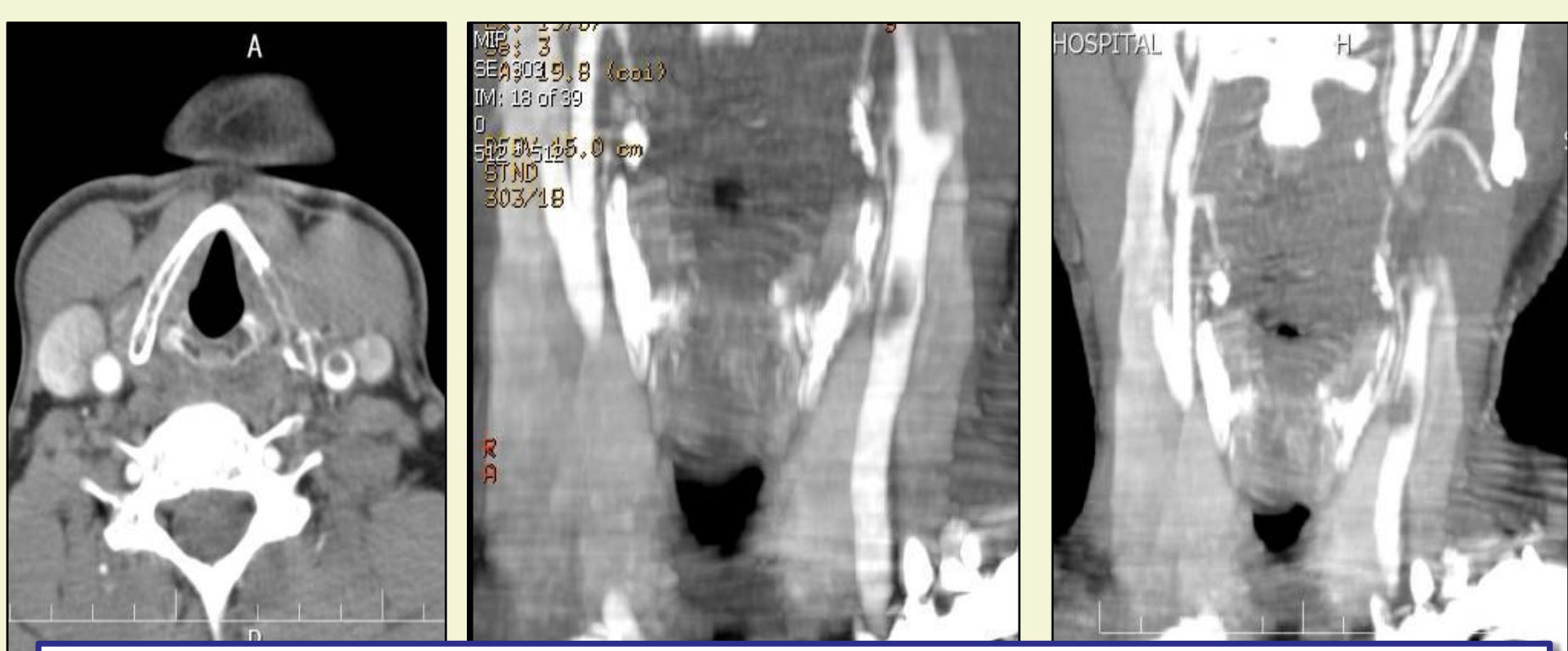

CTA shows a FFT in Lt CCA attached to medial intima, "donut sign" (left). mural attachment of clot (middle), mural attachment plus distal floating part of thrombus, "finger sign" (right).

\section{Conclusions:}

FFT of carotid artery is a rare condition. CCA and carotid bifurcation are the second prevalent sites for FFT after internal carotid artery (ICA). Anemia and reactive thrombocytosis is a very rare cause reported for the condition. In this case, we used DS as screening and CTA to confirm. Since free thrombosis in CCA, can be overlooked, we suggest that every patient with ischemic stroke undergo the adequate investigation to look for FFT. Prompt diagnosis and management is crucial to prevent further ischemic event. Urgent surgery combined with medical management seems the superior approach.

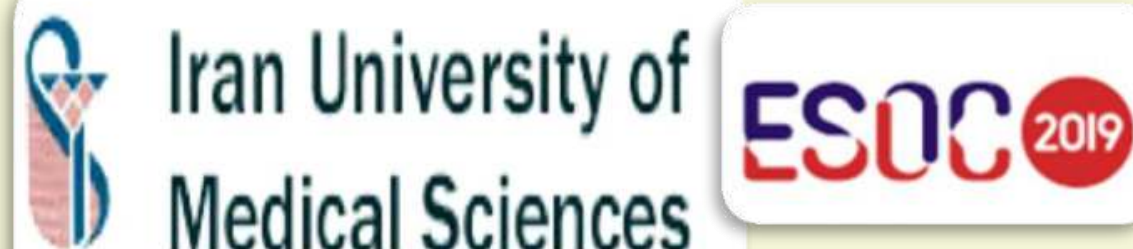

References:

1. Bhatti, Ahmad F., et al. "Free-Floating Thrombus of the Carotid Artery: Literature Review and Case Reports." Journal of Vascular Surgery, vol. 45, no. 1, 2007, pp. 199-205., doi:10.1016/j.jvs.2006.09.057.

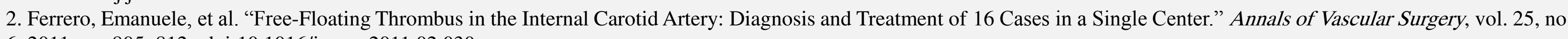
6, 2011, pp. 805-812., doi:10.1016/j.avsg.2011.02.030.

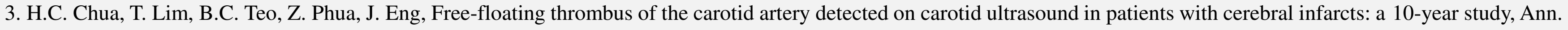
Acad. Med. Singapore 41 (9) (2012) 420-424.

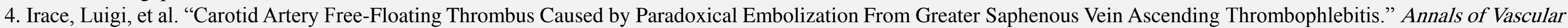
Surgery, vol. 27, no. 4, 2013, doi:10.1016/j.avsg.2012.06.020.

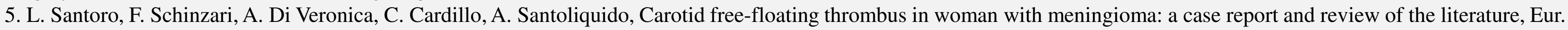
Rev. Med. Pharmacol. Sci. 19 (8) (2015) 1442-1445.

6. Akins, P. T., Glenn, S., Nemeth, P. M., \& Derdeyn, C. P. (1996). Carotid Artery Thrombus Associated With Severe Iron-Deficiency Anemia and Thrombocytosis. Stroke, 27(5), 1002-1005. doi:10.1161/01.str.27.5.1002

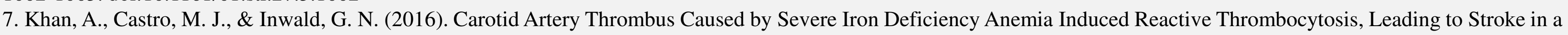
Young Patient. Archives of Physical Medicine and Rehabilitation, 97(12). doi:10.1016/j.apmr.2016.09.045. 